\title{
A hybrid heart for heart failure - how much remains to be done? An interview with Jolanda Kluin
}

\author{
Jolanda Kluin $*, 1$ \\ ${ }^{1}$ Department of Cardiothoracic Surgery, Amsterdam UMC, AMC, Meibergdreef 9, 1105AZ Amsterdam, The Netherlands \\ *Author for correspondence: j.kluin@amsterdamumc.nl
}

\begin{abstract}
Jolanda Kluin speaks to Julia Titova, Commissioning Editor at Future Cardiology.
Jolanda Kluin started her medical training at the Erasmus University Rotterdam in 1989 and obtained her medical degree cum laude in 1996. Thereafter, she worked as a PhD student on the project: "Photodynamic therapy for Barrett's esophagus with use of 5-aminolevulinic acid", receiving her PhD degree cum laude in 1999. In 2006 she finished her residency in cardiothoracic surgery. From 2006 to 2014 she worked as a staff cardiothoracic surgeon at the Department of Cardiothoracic Surgery of the University Medical Center Utrecht and since 2015 she works at the Academic Medical Center, Amsterdam as congenital cardiac surgeon. In 2018 she became a professor in translational cardiothoracic surgery at the University of Amsterdam. Her main interests are grown ups with congenital heart disease cardiac surgery, mitral valve surgery, and reconstructive surgery of the aortic valve and the thoracic aorta. Alongside her clinical tasks, she has started a new line of research in translational cardiothoracic surgery, including tissue engineering of heart valves, valve pathophysiology and the development of a hybrid soft robotic total artificial heart. She is the current chair of the EACTS Women in Cardiothoracic Surgery Committee.
\end{abstract}

First draft submitted: 1 April 2020; Accepted for publication: 1 April 2020; Published online: 23 April 2020

Keywords: artificial heart $\bullet$ congenital heart disease $\bullet$ heart failure

Please tell me about yourself \& your career to date. What first drew you to this area of work $\&$ what is the most rewarding part of your job?

Although I am not from a family of doctors, I knew that I wanted to be a doctor from a very young age. This led me to complete my university studies in medicine with a $\mathrm{PhD}$ in general surgery. I finished my residency in cardiothoracic surgery, and it was here that I found my true calling and I have never looked back since. The other thing that I knew from very early on was that I specifically enjoyed the combination of clinical work and research. I have always worked in a university hospital and particularly enjoy collaborating with people outside the medical field: engineers, physicists, all kinds of people! There are essentially two parts to my current job: the clinical contact with patients and research, both of which are rewarding for different reasons. Helping people on a day-to-day basis and publishing a high-quality research article following months or years of dedication always energizes me!

Your team were recently shortlisted for the British Heart Foundation $£ 30 \mathrm{~m}$ research prize. Could you tell me about the project outlined in your application $\&$ the proposed technology? The aim of this project, which is already ongoing, is to develop a completely artificial soft robotic heart. We call this a hybrid heart. The beating power comes from soft robotics but the inner lining, everything that is in contact with blood, is made from the patients' own cells. There are no 'hard' components in the heart itself so the blood inside the pump only experiences 'soft' materials and the cells from the patient. The energy transfer to power the heart will also be wireless, enabling the patient far greater freedom than currently available. Should we receive this prize, we will be able to develop this idea further, eventually resulting in a clinical implantation of the device.

Future Medicine 


\section{What led you to this idea?}

I have carried out a lot of research on heart valve tissue engineering. What we now aim for is to implant a synthetic biodegradable scaffold in the form of a heart valve. The cells from the patients' own blood enter the scaffold and make their own matrix, eventually degrading the scaffold. This then forms the patients' individual heart valve. From working in this field, I knew that making a real heart from the patients own cells is still far, if we will ever be able to do this.

A few years ago, I saw an interview in a newspaper with a Dutch researcher that contained a picture of a soft robotic octopus [1]. It was then that the idea of making a soft robotic heart first came to me. The combination of my background in making tissue from the patients' cells and the new soft robotic technology gave rise to the idea of a hybrid heart.

\section{At what stage is this research currently? Could you tell me about any preliminary results that have been obtained so far?}

Having proposed this idea, our research team received a grant from the EU, Horizon 2020. From 1 November 2017, we received $£ 3.1$ million to fund this project over the next 5 years. We are now 2 years underway and have since begun to develop a few things. We have one prototype that we are testing in vitro and two in development that have yet to be built. We are eagerly awaiting the publication of our research regarding the beating mechanism over the next few months.

\section{What will the next stage of this research entail?}

In the final year of the EU project, we will be looking to implant the model into a large animal, probably a goat, and we will aim for the hybrid heart to remain in the animal for 3 months with no adverse effects. Should we succeed in our proposal to the British Heart Foundation and receive another $£ 30$ million to fund our project, the aim will be extended to clinical implantation in a patient.

\section{What do you think will be the biggest challenges in translating this to the clinic? How long do you think this will take?}

There will be a range of challenges transferring this research into a clinical situation. A Good Medical Practice certificate is not required for implantation in an animal but will be required to implant this heart in a patient. The additional testing that is required to proceed to clinical testing will need to be funded by companies who can manufacture the device to a Good Medical Practice standard as this cannot be done in our university laboratories. Safety is also a big issue that we will need to address as the failure of the device in any human trial will come at great costs, both financial and the loss of a human life.

On one hand, we must be as safe as possible when experimenting this sort of device. We must ensure that very rigorous testing is carried out prior to human implantation. On the other hand, there are some patients who will die if this device is not made available to them, such as those with end-stage heart failure, for whom heart transplantation may not, for various reasons, be an option. The project we have proposed to the British Heart Foundation will start in 2021 and will last 7 years. We think that within these 7 years, in addition to the 3 years left from the EU grant, we can progress to clinical implantation of the hybrid heart.

\section{What impact do you expect the new hybrid heart will have on cardiac care? Which patients do you think will benefit most from this technology?}

The device will target patients of any age with end-stage heart failure, for example, following myocardial infarction or those with congenital heart disease. The standard of care for these patients is a heart transplant, but due to a shortage of organ donors, this is not an option for many. I do not think this situation will change in the years to come. Left ventricular assist devices, such as HeartMate, are available for those with left ventricular heart disease but are not appropriate for patients with congenital heart disease or right ventricular heart failure. These devices also come with a lot of complications and low quality of life.

In the Netherlands, we do not have access to a completely artificial heart. SynCardia Total Artificial Heart is the only US FDA-approved device, but it is not available here, meaning patients with biventricular failure must travel abroad to have it fitted. The drawbacks of this device are similar to the limitations of left ventricular assist devices, although complications arising from SynCardia Total Artificial Heart are even more frequent and severe. 
The quality of life is also significantly affected. These are some of the things that we aim to change by developing the soft robotic heart.

It is difficult to say who the first patient may be. Carrying out such an operation in a young child is difficult from an ethical and a technical point of view. Therefore, I suspect our first patient will be an older patient who is able to make an informed decision in order to provide consent for such a procedure. Eventually, this new technology may be used in all cardiac patients who require heart transplantation.

\section{Could this technology be applied to other organs \& diseases?}

The heart is ideal in the sense that its only function is that of a pump. The difficulty with providing a similar system for other organs such as the lungs, kidney or liver, is that they also have an additional metabolism function. It is therefore much more challenging as multiple types of cells are required. For muscles, such as those in legs, there may be a possibility to develop a similar system and could be an area of research in the future.

\section{What one piece of advice would you give to younger researchers \& clinicians regarding balancing their work \& social lives?}

It is very difficult, I agree! The best advice would be to avoid spending time working on something that does not energize you. Life at home and at work should be exciting, even thrilling! We all have day-to-day obligations, such as administrative activities, but I try to schedule these around the things that you enjoy, so that there is something special to look forward to every day.

\section{Financial \& competing interests disclosure}

The projects discussed in this interview have received funding from the European Union's Horizon 2020 research and innovation programme under grant agreement No 767195 and the Netherlands Cardiovascular Research Initiative (CVON 2012-01): The Dutch Heart Foundation, Dutch Federation of University Medical Centers, the Netherlands Organization for Health Research and Development and the Royal Netherlands Academy of Sciences. The author has no other relevant affiliations or financial involvement with any organization or entity with a financial interest in or financial conflict with the subject matter or materials discussed in the manuscript apart from those disclosed.

No writing assistance was utilized in the production of this manuscript.

\section{Disclaimer}

The opinions expressed in this interview are those of the interviewee and do not necessarily reflect the views of Future Medicine Ltd.

\section{Reference}

1 NRC Handelsblad, Octopus als robot (2016). www.nrc.nl/nieuws/2016/09/02/octopus-als-robot-4091564-a1519493 
